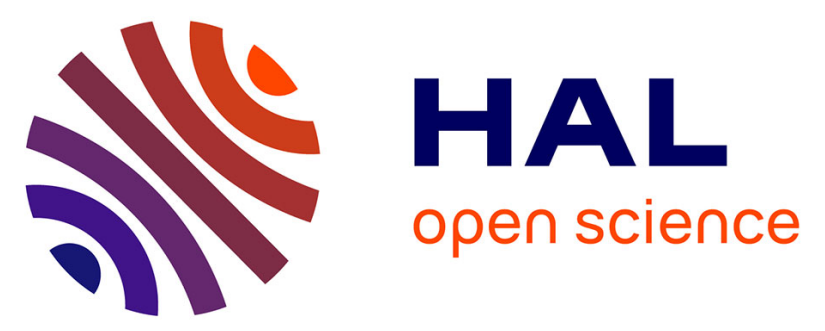

\title{
Nonlinear optical susceptibility described with a spherical formalism applied to coherent anti-Stokes Raman scattering
}

Carsten Cleff, Herve Rigneault, Sophie Brasselet, Julien Duboisset

\section{- To cite this version:}

Carsten Cleff, Herve Rigneault, Sophie Brasselet, Julien Duboisset. Nonlinear optical susceptibility described with a spherical formalism applied to coherent anti-Stokes Raman scattering. Physical Review A : Atomic, molecular, and optical physics [1990-2015], 2017, 96 (1), pp.013851. 10.1103/PhysRevA.96.013851 . hal-01569630

\section{HAL Id: hal-01569630 \\ https://hal.science/hal-01569630}

Submitted on 12 Apr 2018

HAL is a multi-disciplinary open access archive for the deposit and dissemination of scientific research documents, whether they are published or not. The documents may come from teaching and research institutions in France or abroad, or from public or private research centers.
L'archive ouverte pluridisciplinaire HAL, est destinée au dépôt et à la diffusion de documents scientifiques de niveau recherche, publiés ou non, émanant des établissements d'enseignement et de recherche français ou étrangers, des laboratoires publics ou privés. 


\title{
Nonlinear optical susceptibility described with a spherical formalism applied to coherent anti-Stokes Raman scattering
}

\author{
Carsten Cleff, Hervé Rigneault, Sophie Brasselet, and Julien Duboisset* \\ Aix-Marseille Université, CNRS, Centrale Marseille, Institut Fresnel, UMR 7249, 13397 Marseille, France
}

(Received 15 May 2017; published 25 July 2017)

\begin{abstract}
We describe coherent Raman scattering in a complete spherical formalism allowing a better understanding of the coherent Raman process with respect to its symmetry properties, which is especially helpful in polarized coherent Raman microscopy. We describe how to build the coherent Raman tensor from spontaneous Raman tensor for crystalline and disordered media. We introduce a distribution function for molecular bonds and show how this distribution function results in a new macroscopic symmetry which can be very different from the symmetry of vibrational modes. Finally, we explicitly show polarization configurations for coherent anti-Stokes Raman scattering to probe specific vibration symmetries in crystalline samples and lipid layers.
\end{abstract}

DOI: 10.1103/PhysRevA.96.013851

\section{INTRODUCTION}

Since several years, coherent anti-Stokes Raman scattering (CARS) microscopy has attracted much interest because of its capabilities for label free, three-dimensional, chemical selective, and real-time imaging [1-3]. The growing interest in nonlinear microscopy has also resulted in important efforts towards polarization-resolved measurements to recover orientation and symmetries of molecules in the focal volume [4-8]. The CARS susceptibility tensor, which describes how molecules will respond to various incoming field polarization states, is a third-rank tensor and possesses a priori more information than the first-rank susceptibility tensor associated to spontaneous Raman. As a consequence, CARS tensor together with the coherent nature of the CARS process have the potential to provide additional valuable information about sample chemical orientations and organizations. However, it also results in a higher complexity and, together with the contribution of the CARS nonresonant background, the interpretation of CARS polarization measurements to retrieve molecular information can be difficult [6,9-11].

The analysis of experimental results can be eased using a different formalism. Instead of using Cartesian coordinates, one can use the spherical formalism, known also as irreducible formalism. The spherical formalism allows to describe a tensor in a basis where its components remain unchanged under rotation [12-15], providing a more suitable framework for an investigation of symmetry properties. The spontaneous Raman scattering tensors for the 32 crystallographic groups have already been expressed as function of spherical components by Gächter [16]. However, the link between the crystallographic groups and the CARS tensor is still missing and has never been discussed before in the spherical formalism framework. Recently, we demonstrated experimental results on the direct measurement of the spherical components of Raman and CARS tensors using circular polarization for the excitation and detection [17], providing new possibilities to recover symmetry properties of molecular assemblies.

The objective of this work is to derive a spherical-based formalism which describes CARS susceptibilities in a very

\footnotetext{
*julien.duboisset@fresnel.fr
}

general context. To achieve this, (1) the Cartesian tensor is expressed as function of its spherical components, (2) the spherical components of the CARS tensor are expressed as a function of spontaneous Raman spherical components, and (3) the orientation and the distribution of molecules in the focal volume are taken into account to calculate the macroscopic CARS susceptibility observed within the focal volume. Finally, we explain how the spherical CARS components can be directly measured using a specific set of polarization states, and we experimentally demonstrate this on crystal sample and disordered biological sample.

\section{THEORY}

\section{A. Spherical components of third-rank tensor}

We assume that the electronic dipole moments induced in four-wave mixing processes can be written as

$$
P_{h}=\epsilon_{0} \gamma_{h i j k} E_{i} E_{j} E_{k},
$$

where $P$ is the dipole moment, $\gamma$ is the third-rank susceptibility tensor with 81 components, $E$ are the electric fields, and $h, i, j, k$ represent the Cartesian coordinates $x, y, z$. The important limitation of the Cartesian frame is the dependence of the tensor components' values on the direction axes, whereas physical properties are intrinsic matter properties that are not affected by rotations of the coordinate system. Therefore, often the concept of rotational invariant is used to describe the properties of matter, independently of the coordinate system. This can be done by decomposing the $\gamma$ tensor into spherical parts. Any $n$-rank Cartesian tensor can be written as the direct sum of spherical tensor, each labeled by an order $l$ (an integer equal or smaller than $n+1$ ) and having $m=2 l+1$ independent components which transform under rotation, whereas the order $l$ remains unchanged. First, it is necessary to establish a transformation between Cartesian and spherical tensors. We derive the transformation for a third-rank tensor in a similar way as it was done for a second-rank tensor [12,18]. The components $\gamma_{m}^{l}$, expressing the nonlinear tensor in the spherical functions basis $Y_{m}^{l}$, are connected to the $\gamma_{h i j k}$ components, expressing the nonlinear tensor in Cartesian 
TABLE I. Unitary third-rank tensor as function of its Cartesian and spherical components.

\begin{tabular}{|c|c|c|c|c|c|c|c|c|c|}
\hline $\begin{array}{l}C_{ \pm m}^{l} \\
C_{h i j k}\end{array}$ & $\begin{array}{l}\text { वै } \\
4: \pm 4\end{array}$ & $\begin{array}{c}d b \\
4 ; \pm 3\end{array}$ & $\begin{array}{c}\text { d } \\
4 ; \pm 2\end{array}$ & $\begin{array}{c}\mathscr{U} \\
4 ; \pm 1\end{array}$ & $\begin{array}{c}l \\
4 ; 0\end{array}$ & $\begin{array}{c}0 \\
2 ; \pm 2\end{array}$ & $\begin{array}{c}\infty \\
2 ; \pm 1\end{array}$ & $\begin{array}{c}8 \\
2 ; 0\end{array}$ & $0 ; 0$ \\
\hline & & & & & & & & & \\
\hline$x \times x x$ & $1 / 4$ & & $-1 / \sqrt{28}$ & & $\begin{array}{l}3 / \sqrt{280} \\
3 / \sqrt{280}\end{array}$ & $-\sqrt{3 / 14}$ & & $\begin{array}{l}1 / \sqrt{7} \\
1 / \sqrt{7}\end{array}$ & $\begin{array}{l}1 / \sqrt{5} \\
1 / \sqrt{5}\end{array}$ \\
\hline yyyy & $1 / 4$ & & $1 / \sqrt{ } 28$ & & $3 / \sqrt{280}$ & $\sqrt{3 / 14}$ & & $1 / \sqrt{7}$ & $1 / \sqrt{5}$ \\
\hline$x x y y$ & $-1 / 4$ & & & & $1 / \sqrt{280}$ & & & $1 / \sqrt{ } 63$ & $1 / \sqrt{ } 45$ \\
\hline$x x x y$ & $\pm i / 4$ & & $\mp i / \sqrt{112}$ & & & $\mp i \sqrt{3 / 56}$ & & & \\
\hline xyyy & $\mp i / 4$ & & $\mp i / \sqrt{112}$ & & & $\mp i \sqrt{3 / 56}$ & & & \\
\hline$x x z z$ & & & $1 / \sqrt{28}$ & & $-\sqrt{2 / 35}$ & $-1 / \sqrt{168}$ & & $-1 / \sqrt{252}$ & $1 / \sqrt{45}$ \\
\hline yyzz & & & $-1 / \sqrt{28}$ & & $-\sqrt{2 / 35}$ & $1 / \sqrt{168}$ & & $-1 / \sqrt{252}$ & $1 / \sqrt{45}$ \\
\hline$x y z z$ & & & $\pm i / \sqrt{28}$ & & & $\mp i / \sqrt{168}$ & & & \\
\hline$x x x z$ & & $\mp 1 / \sqrt{32}$ & & $\pm 3 / \sqrt{224}$ & & & $\pm \sqrt{3 / 56}$ & & \\
\hline yyyz & & $i / \sqrt{32}$ & & $3 i / \sqrt{224}$ & & & $i \sqrt{3 / 56}$ & & \\
\hline$x x y z$ & & $-i / \sqrt{32}$ & & $i / \sqrt{224}$ & & & $i / \sqrt{168}$ & & \\
\hline$x y y z$ & & $\pm 1 / \sqrt{32}$ & & $\pm 1 / \sqrt{224}$ & & & $\pm 1 / \sqrt{168}$ & & \\
\hline$z z z x$ & & & & $\mp 1 / \sqrt{14}$ & & & $\pm \sqrt{3 / 56}$ & & \\
\hline$z z z y$ & & & & $-i / \sqrt{14}$ & & & $i \sqrt{3 / 56}$ & & \\
\hline$z z z z$ & & & & & $\sqrt{8 / 35}$ & & & $-\sqrt{4 / 7}$ & $1 / \sqrt{5}$ \\
\hline
\end{tabular}

coordinates, according to the following relation:

$$
\gamma_{m}^{l}=\sum_{h i j k} C_{h i j k}^{l, m} \gamma_{h i j k},
$$

where $C_{h i j k}^{l, m}$ is the transformation matrix between Cartesian and spherical basis and is expressed explicitly in Table I. Details on how $C_{h i j k}^{l, m}$ is calculated can be found in the Appendix.

Conversely, the passage to the Cartesian form of the tensor is possible by using the irreducible tensor basis $\bar{C}_{m}^{l}$

$$
\bar{\gamma}_{\text {Cart }}=\sum_{\oplus} \gamma_{m}^{l} \bar{C}_{m}^{l} .
$$

The spherical formalism allows to describe a nonlinear tensor as a sum of spherical harmonics functions with useful symmetry properties. Therefore, the symmetry-based analysis of a four-wave mixing process is easier and allows to describe the nonlinear tensor with fewer components than in Cartesian coordinates.

\section{B. CARS spherical components for crystallographic point groups}

Coherent anti-Stokes Raman scattering is a stimulated Raman process as well as a nonlinear four-wave mixing process. In the CARS process a photon, called pump, at $\omega_{p}$ and a photon called Stokes at $\omega_{s}$ interact with a molecule and excite a vibrational resonance at $\Omega=\omega_{p}-\omega_{s}$. A photon, called probe, at $\omega_{p r}$ interacted with the excited vibration resonance of the molecule generating a new blue-shifted photon, called anti-Stokes at $\omega_{p}+\omega_{p r}-\omega_{s}$. Experimentally, often degenerated CARS is performed where the pump and probe frequencies are the same.

The CARS process is described by two Raman interactions, one Stokes (excitation of Raman state) and one anti-Stokes (probing of Raman state) $[19,20]$. Thus, the CARS tensor can be described by a tensorial product of two Raman tensors, the
Stokes tensor $\bar{\alpha}_{s}$ and the anti-Stokes tensor $\bar{\alpha}_{a s}$ :

$$
\bar{\gamma}_{\mathrm{CARS}}=\bar{\alpha}_{s} \otimes \bar{\alpha}_{a s},
$$

where $\otimes$ represents the tensorial product. Whereas the Cartesian calculation of the CARS tensor, from the Raman tensor, is tedious [10], the calculation in the spherical basis is straightforward. In the absence of one-photon resonance or competitive two-photon process, the spherical components of $\bar{\gamma}$ are

$$
\gamma_{m}^{l}=\sum_{l_{1}, l_{2}}\left(l_{1} m_{1} l_{2} m_{2} \mid l m\right) \alpha_{m_{1}}^{l_{1}} \alpha_{m_{2}}^{l_{2}}
$$

where $\left(l_{1} m_{1} l_{2} m_{2} \mid l m\right)$ are the Clebsch-Gordan coefficients which impose the rules $m=m_{1}+m_{2}$ and $\left|l_{1}-l_{2}\right| \leqslant l \leqslant l_{1}+$ $l_{2}$. Consequently, the spherical components of the $\gamma$ CARS tensor is the product of the spherical components of the Raman tensor, weighted by the Clebsch-Gordan coefficient. The Raman tensor in the spherical basis was already investigated for each group of symmetry by Gächter [16] (see Table II).

The Raman and CARS susceptibilities for a given vibrational mode, respectively $\bar{\alpha}$ and $\bar{\gamma}$, are therefore described by spherical components. In the most general case, each mode of vibration is represented by an infinite number of spherical components [21]. However, the first-rank tensor of Raman susceptibility operates as truncation and its spherical components can not exceed $l \leqslant 2$ and $-l \leqslant m \leqslant l$, whatever the symmetry of the vibration modes. For instance, the symmetric vibration $A_{1 g}$ of the group $O_{h}$ is, according to Gächter, made by spherical components of order $\alpha_{0}^{0}$. According to the CARS scheme, the tensorial product of two tensors of order $0, \alpha_{0}^{0}$ leads to unique components of $\gamma_{0}^{0}$. Similarly, the antisymmetric vibration $T_{2 g}$ possesses $\alpha_{2}^{2}$ spherical components which lead to only one spherical component $\gamma_{4}^{4}$ (see Table II). Thus, the prediction of spherical components of CARS tensor is direct and easy using spherical formalism. 
TABLE II. Spherical components of the first- and third-rank susceptibility tensors as function of two different point-group symmetries. When a mode is degenerated, the different contribution appears on different lines. The components in parentheses are inactive using in-plane polarization fields.

\begin{tabular}{|c|c|c|c|}
\hline & & \multicolumn{2}{|c|}{ Spherical components } \\
\hline \multicolumn{2}{|c|}{ Group } & Raman [16] & Coherent Raman \\
\hline \multirow[t]{6}{*}{$O_{h}$} & $A_{1 g}$ & $\alpha_{0}^{0}$ & $\gamma_{0}^{0}$ \\
\hline & $F$ & $\alpha_{0}^{0}$ & $\gamma_{0}^{0}$ \\
\hline & $\boldsymbol{E}_{g}$ & $\alpha_{2}^{2}$ & $\gamma_{4}^{4}$ \\
\hline & $T_{1 g}$ & $\left(\alpha_{1}^{1}\right)$ & \\
\hline & & $\left(\alpha_{0}^{1}\right)$ & \\
\hline & $T_{2 g}$ & $\begin{array}{c}\alpha_{2}^{2} \\
\left(\alpha_{1}^{2}\right)\end{array}$ & $\gamma_{4}^{4}$ \\
\hline \multirow{4}{*}{$D_{2}$} & $A$ & $\alpha_{0}^{0}, \alpha_{0}^{2}, \alpha_{2}^{2}$ & $\gamma_{0}^{0}, \gamma_{0}^{2}, \gamma_{2}^{2}, \gamma_{2}^{4}, \gamma_{4}^{4}$ \\
\hline & & $\alpha_{0}^{0}, \alpha_{0}^{2}$ & $\gamma_{0}^{0}, \gamma_{0}^{2}, \gamma_{0}^{4}$ \\
\hline & $B_{1}$ & $\left(\alpha_{0}^{1}\right), \alpha_{2}^{2}$ & $\gamma_{4}^{4}$ \\
\hline & $B_{2}, B_{3}$ & $\left(\alpha_{1}^{1}\right),\left(\alpha_{1}^{2}\right)$ & - \\
\hline
\end{tabular}

\section{Macroscopic CARS susceptibility tensor}

The macroscopic susceptibility tensor in the laboratory frame $\bar{\chi}$ usually differs from the microscopic susceptibility tensor $\bar{\gamma}$ itself due to the collective orientation of the molecules in the probed focal volume. In a crystal, the macroscopic susceptibility tensor is similar to the microscopic molecular tensor. In a more disordered media, one has to introduce an angular distribution function, which represents the probability to find a molecule pointing in a certain direction and there is no direct link between the macroscopic and microscopic susceptibility tensors as found in crystal [5]. To build the macroscopic susceptibility tensor in the laboratory frame, one has to convolve the microscopic susceptibility tensor by the distribution function

$$
\begin{aligned}
\chi= & \frac{1}{(2 \pi)^{2}} \int_{0}^{2 \pi} \int_{0}^{\pi} f(\theta, \varphi) \\
& \times \gamma\left(\theta-\theta_{0}, \varphi-\varphi_{0}\right) \sin \theta d \theta d \varphi,
\end{aligned}
$$

where $\theta_{0}$ and $\varphi_{0}$ are the molecule angles in the microscopic frame. The distribution function $f(\theta, \varphi)$ can be also described as a series of spherical harmonics

$$
f(\theta, \varphi)=\sum_{l, m} f_{m}^{l} Y_{m}^{l}(\theta, \varphi),
$$

where the coefficients $f_{m}^{l}$ are the components of the spherical basis functions $Y_{m}^{l}(\theta, \varphi)$. The spherical harmonic convolution theorem tells that the spherical components of the convolution product are simply the product of the same spherical component of each term [22], thus,

$$
\chi_{m}^{l}=\gamma_{m}^{l} f_{m}^{l}
$$

Therefore, the spherical component in the laboratory frame is the product between the same order spherical components of the microscopic CARS susceptibility and the molecular distribution function (see Fig. 1). In the special case of a crystal, the distribution function is a delta function $\delta\left(\theta_{0}, \varphi_{0}\right)$ which implies that all the $f_{m}^{l}$ are not vanishing. Then, the

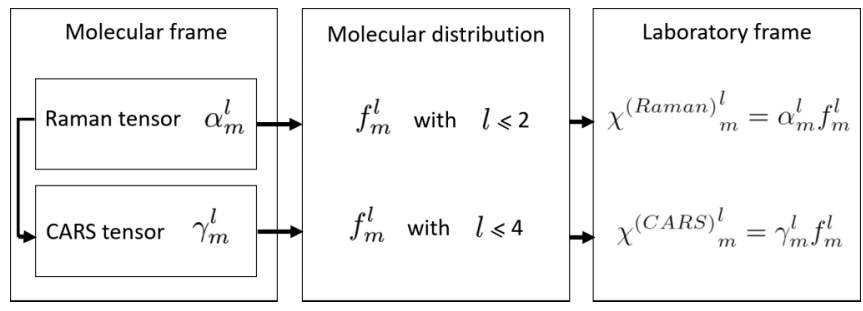

FIG. 1. General process for the passage from microscopic tensor to macroscopic tensor with the spherical formalism.

macroscopic tensor is equal to the microscopic tensor. On the contrary, in a liquid solution the molecules show all possible orientations and the distribution function contains only one not vanishing term, the isotropic one $f_{0}^{0}$, which filters the microscopic susceptibility leading to the macroscopic one $\chi_{0}^{0}$ [12]. When the molecules are partially organized within the focal volume, somewhat between a crystal and a liquid, the distribution function does not possess all the orders, especially the high orders, and Eq. (8) operates as a filtering process of the molecular symmetry.

\section{Measurement of spherical components with circular polarization}

To be consistent with the spherical description of the susceptibility tensor $\bar{\chi}$, we also describe the electric fields involved in the CARS process as spherical harmonic functions. All the polarization dependence in Eq. (1) can be collected in a unique tensor $\bar{F}$ defined by

$$
\begin{aligned}
p & =\epsilon_{0} \bar{\chi} \cdot\left[\hat{e}_{a s}^{*} \otimes \hat{e}_{p} \otimes \hat{e}_{s}^{*} \otimes \hat{e}_{p r}\right] \\
& =\epsilon_{0} \bar{\chi} \cdot \bar{F},
\end{aligned}
$$

where the subscripts $\hat{e}_{p}, \hat{e}_{s}^{*}, \hat{e}_{p r}, \hat{e}_{a s}^{*}$ refer to electric field polarization of anti-Stokes, pump, Stokes, and probe beam respectively, and the asterisk stands for the complex conjugate. The electric field polarization expressed in the Cartesian frame can be also expressed into the spherical formalism. A vector is a rank-zero tensor and consequently can be decomposed onto spherical components of $l=1$. In the spherical basis, the leftand right-handed circular polarization and linear $z$-polarization states correspond to spherical components of the electric field $e_{m=1}^{l=1}, e_{m=-1}^{l=1}$, and $e_{m=0}^{l=1}$, respectively $[17,18]$. Written in the spherical basis, the spherical components of the tensor field are

$$
\bar{F}_{m_{F}}=\sum_{l} k^{l} e_{m_{a s}}^{* 1} e_{m_{p}}^{1} e_{m_{s}}^{* 1} e_{m_{p r}}^{1},
$$

where $k^{l}$ is a coefficient which depends only of the $l$ value, linked to Clebsch-Gordan coefficients [6], and the resulting $m_{F}$ value being a summation of field's $m$ values

$$
m_{F}=m_{p}-m_{s}+m_{p r}-m_{a s},
$$

paying attention to the presence of conjugated fields in the nonlinear processes, which imply a change of the $m$ sign (since $\hat{e}_{m}^{* l}=\hat{e}_{-m}^{l}$ ). Therefore, the light field tensor $\bar{F}$ has a $m_{\bar{F}}$-rotational symmetry in the sample plane defined by the $m$ values of the individual incident and emitted light fields. 

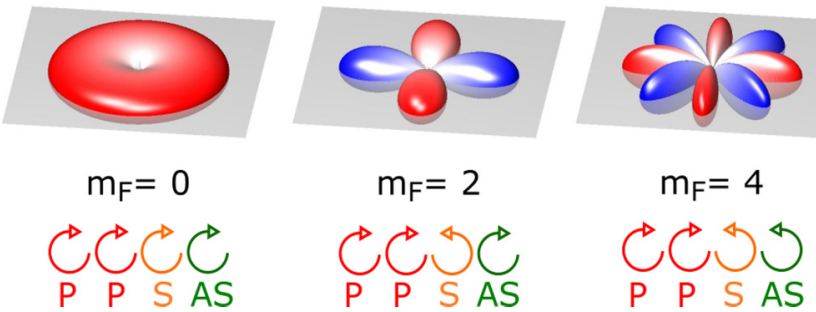

FIG. 2. Spherical tensors generated with three different circular polarization schemes according to Eq. (12). $m_{F}=0$ is read out when all the fields have the same circular polarization and the order $m_{F}=2$ when the Stokes or the anti-Stokes beam have the opposite polarization handiness with respect to the pump beam. $m_{F}=4$ is read when the Stokes and the anti-Stokes polarizations have the opposite handiness with respect to the pump.

Using Eqs. (10), (11), and (9), it can be seen that degenerated CARS with circular polarization for pump, Stokes, and probe allows a direct read-out of sample symmetries of order 0 (isotropic, $\left.m_{\bar{F}}=0\right)$, order $2\left(m_{\bar{F}}=2\right)$, and order 4 $\left(m_{\bar{F}}=4\right)$ determined by the chosen field configurations, creating a symmetry-based contrast mechanism [17]. According to Eq. (11), the order 0 is read out when all the fields have the same circular polarization and the order 2 when the Stokes or the anti-Stokes beam have the opposite polarization handiness with respect to the pump beam. Order 4 is read when the Stokes and the anti-Stokes polarizations have the opposite handiness with respect to the pump. Using Eq. (10) and the values of $k^{l}$ expressed in [23], the related field tensors expressed into the spherical basis are

$$
\begin{aligned}
& \bar{F}_{m_{F}=4} \propto \bar{C}_{4}^{4}, \\
& \bar{F}_{m_{F}=2} \propto \sqrt{1 / 28} \bar{C}_{2}^{4}-\sqrt{3 / 14} \bar{C}_{2}^{2}, \\
& \bar{F}_{m_{F}=0} \propto \sqrt{1 / 70} \bar{C}_{0}^{4}-\sqrt{4 / 63} \bar{C}_{0}^{2}+\sqrt{4 / 45} \bar{C}_{0}^{0},
\end{aligned}
$$

where $\bar{C}_{m}^{l}$ is the unitary spherical tensor of order $l$ and $m$ described in Table I. Another way to find this result is to write the electric field polarization in the spherical basis $\theta, \varphi$. The in-plane circular polarizations are

$$
\begin{gathered}
e_{1}^{1} \propto \sin \theta e^{i \varphi}, \\
e_{-1}^{1} \propto \sin \theta e^{-i \varphi},
\end{gathered}
$$

where $\theta$ is the angle with respect to the propagation axis $z$ and $\varphi$ is the angle in the $x y$ plane. Consequently, the product of four electric fields involved in the constitutive equation (9) generates a field function $F(\theta, \varphi)$ which leads to the same result as Eq. (12) and corresponds to the result illustrated in Fig. 2.

The intensity scattered by an assembly of molecules can thus be expressed in terms of spherical tensor components $\chi_{m}^{l}$ and $F_{m}^{l}$. For a coherent process, the scattered intensity is

$$
\begin{aligned}
I_{\mathrm{coh}} & =A(V, k) \sum_{l}(-1)^{l}\left(\chi_{m_{F}}^{l}\right)^{2}\left(F_{-m_{F}}^{l}\right)^{2} \\
& =A(V, k) \xi_{m_{F}}^{2}
\end{aligned}
$$

with

$$
\xi_{m_{F}}=\sum_{l}(-1)^{l} \chi_{m_{F}}^{l} k^{l}
$$

where $\xi_{m_{F}}$ can be interpreted as an effective susceptibility with an in-plane $m_{F}$ symmetry order, which encompasses the full $\chi_{m}^{l}$ components by a summation over $l . A(V, \vec{k})$ is a term proportional to the molecular density and involves phase-matching conditions [24].

\section{EXPERIMENTS}

\section{A. Methods}

\section{CARS experimental setup}

CARS imaging was performed on a custom-built microscope setup incorporating a picosecond dual color laser system [25]. The laser system is composed of two optical parametric oscillators (OPO1 and OPO2, Emerald, APE) synchronously pumped by a mode-lock frequency doubled Nd:YVO Laser (PicoTrain, HighQLaser) operating at $532 \mathrm{~nm}$. The two mode-locked beams from OPO1 (pump) and OPO2 (Stokes) (pulse duration $5 \mathrm{ps}$, repetition rate $76 \mathrm{MHz}$ ) are overlapped in time and space and sent into a custom-made scanning microscope. For spectral scanning, the pump wavelength is fixed to $730 \mathrm{~nm}$ and the Stokes wavelength is scanned in the spectral windows from 775 to $800 \mathrm{~nm}$ and 870 to $885 \mathrm{~nm}$ in order to excite vibrational resonances from 795 to $1200 \mathrm{~cm}^{-1}$ and from 2200 to $2400 \mathrm{~cm}^{-1}$. The CARS signal is detected in forward direction using PMTs (Hamamatsu, H10682) working in the photon-counting regime. Excitation and collection are performed using two identical NA $=0.6$ objectives (Olympus UCPlan FL 40x). The incident powers at the sample plane were 1-4 $\mathrm{mW}$ for the pump beam and 1-5 $\mathrm{mW}$ for the Stokes beam depending on the samples. Imaging is performed by scanning galvanometric mirrors (typically: pixel dwell time of $50 \mu \mathrm{s}$, $100 \times 100$ pixels, and scan range of $30 \mu \mathrm{m})$. A home-built software controls the galvo mirrors, data acquisition, and OPO wavelength tuning [26]. In order to achieve symmetry-resolved CARS (SR-CARS) imaging, an achromatic quarter-wave plate was inserted before the focusing microscope objective to excite the sample with circularly polarized light. The linear polarization state (before the quarter-wave plate) of the Stokes beam was switched between $\mathrm{V}$ and $\mathrm{H}$ polarization, resulting in a switching between left- and right-handed circular polarization states in the sample plane. The generated CARS signal passed through a second achromatic quarter-wave plate converting circular polarization to linear polarization. Subsequently, a Wollaston prism split the CARS beam into $\mathrm{V}$ and $\mathrm{H}$ polarization, which were detected individually with photomultiplier tubes [17]. On the same microscope, Raman spectra were acquired using a HeNe laser at $632.8 \mathrm{~nm}$ and a spectrograph (Horiba iHR320) equipped with a Peltier-cooled CCD detector.

\section{Zeolite crystal samples}

The first sample studied in this work is an octahydrosilasesquioxane $\mathrm{H}_{8} \mathrm{Si}_{8} \mathrm{O}_{12}$ crystal (HT8), that has cubic symmetry and belongs to the $\mathrm{O}_{h}$ crystallographic point group. The $\mathrm{H}_{8} \mathrm{Si}_{8} \mathrm{O}_{12}$ crystal synthesis can be found in [27]. For CARS spectroscopy, micrometric to millimetric size 
(a)

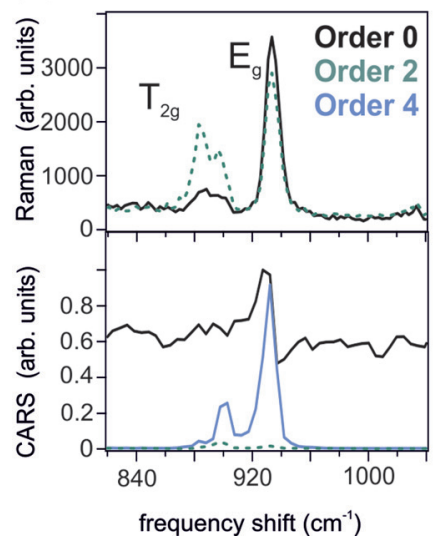

(b)

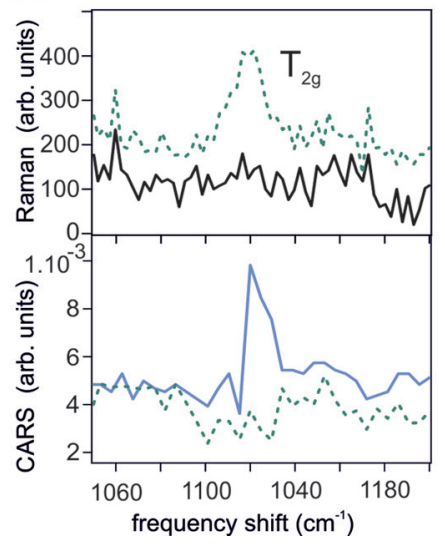

(c)

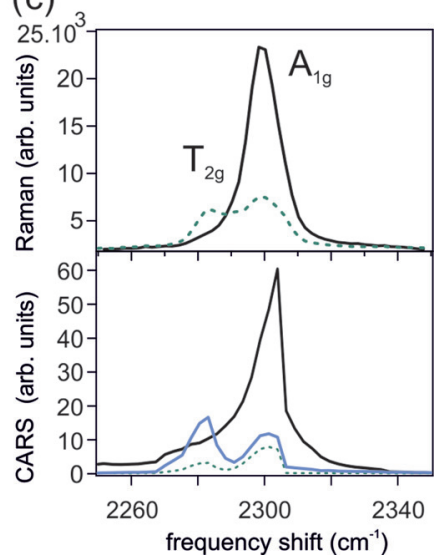

FIG. 3. Symmetry-resolved CARS and spontaneous Raman spectrum of HT8 zeolite crystal. (a), (b) $T_{2 g}$ and $E_{g}$ resonance in the $O-H$ bending mode region. (c) $T_{2 g}$ and $A_{1 g}$ in the $O-H$ stretching mode region. The order 0 is the black line, order 2 the dashed green line, and order 4 the blue (gray) line.

crystals were directly deposited onto microscope coverslip and surrounded by water.

\section{Multilamellar vesicle samples}

As a second sample, multilamellar vesicle samples (MLVs) were made from chain 1,2-dipalmitoyl-sn-glycero-3phosphocholine (DPPC) lipids and 5\% cholesterol. DPPC was hydrated in phosphate-buffered saline [(PBS), $\mathrm{pH} 7.4]$ above the main phase-transition temperature $\left(45^{\circ} \mathrm{C}\right)$ for $1 \mathrm{~h}$, leading to MLVs of $1-30 \mu \mathrm{m}$ size in a solution enclosed between two spaced coverslips. The MLVs form almost spherical objects made of concentric multilayers of lipids. Imaging was performed at the equatorial plane of these objects where the distribution of the lipids is expected to be oriented in the transverse sample plane.

\section{B. Results}

\section{Zeolite crystal}

A crystalline sample offers an ideal starting point to verify the link between the Raman and CARS susceptibilities since the microscopic tensor is the same as the macroscopic tensor. The HT8 zeolite crystal is a cubic molecular crystal which offers vibrational resonances belonging to a variety of symmetries. In a first step we investigate the three main Raman active symmetries $A_{1 g}, E_{g}$, and $T_{2 g}$ using circular polarization spontaneous Raman spectroscopy (see Fig. 3). As predicted by Gätcher, the $A_{1 g}$ strong resonance at $2300 \mathrm{~cm}^{-1}$ is mainly present on the order zero. The measured order 2 contribution is attributed to polarization leakage since no order two is expected at $2300 \mathrm{~cm}^{-1}$. The $T_{2 g}$ resonances, at 910 and $1120 \mathrm{~cm}^{-1}$, are only present on the order 2 . The $E_{g}$ resonance at $932 \mathrm{~cm}^{-1}$ appears on both order 0 and order 2 .

Symmetry-resolved CARS shows a different signature than spontaneous Raman. The $A_{1 g}$ resonance is mainly composed of an order- 0 contribution. The order- 2 and -4 contributions are 10 times less intense and most likely artifacts of polarization leakage. The $T_{2 g}$ resonances have a clear order-4 symmetry while the $E_{g}$ resonance shows symmetry components of order 0 and order 4 . It can be seen that the order- 0 contribution interferes with the nonresonant CARS signal of the zeolite which is mainly of order 0 resulting in the typical CARS line shape and shift of the peak resonance.

The different orders of symmetry read out by the CARS and the Raman follow our explanation by the tensorial product of two Raman tensors, as demonstrated in the theoretical part. The CARS susceptibility is thus the tensorial product of two Raman tensors that are intrinsically limited to order 2 and, consequently, the CARS tensor does not possess more information than the first-rank tensor.

\section{Multilamellar vesicles (MLV)}

DPPC multilamellar vesicles represent a different kind of sample than crystalline sample as they show a macroscopic symmetry different to the symmetry of their microscopic lipid building blocks. They are interesting samples as they offer a large variety of symmetries and controlled packing conditions when mixed with cholesterol, allowing to monitor different distribution function [28]. Symmetry-resolved CARS spectra were acquired between 1030 and $1180 \mathrm{~cm}^{-1}$, to observe the symmetric and antisymmetric bending of the lipid carbon-carbon bond. The antisymmetric bending at $1060 \mathrm{~cm}^{-1}$ and the symmetric bending at $1133 \mathrm{~cm}^{-1}$ are known to be $A$ resonances of the group $D_{2 h}$. The difference between these two vibrations is only the different ratio between the order- 0 and the order- 2 contributions [29]. The antisymmetric bending contains a higher order- 2 than order- 0 contribution while in the symmetric bending it is the other way around. For a $D_{2 h}$ resonance, the CARS susceptibility of the antisymmetric bending should contain order- 0 , order-2, and order 4-contributions [see Table II and Eq. (4)]. However, while we observe order-0 and order- 2 contributions, the order- 4 contribution is absent for both resonances [see Fig. 4(a)]. The deviation between our measurement and the prediction for a pure $D_{2 h}$ resonance results from the smooth distribution function by which the microscopic building blocks are oriented to each other. Due to the loose angular correlation of the lipid building blocks, the order 4 of the $D_{2 h}$ resonance gets filtered out when averaging over many of them within the focal volume. In the case where multilamellar vesicles are made with $5 \%$ cholesterol, the packing of molecules is more rigid, leading to a sharper 

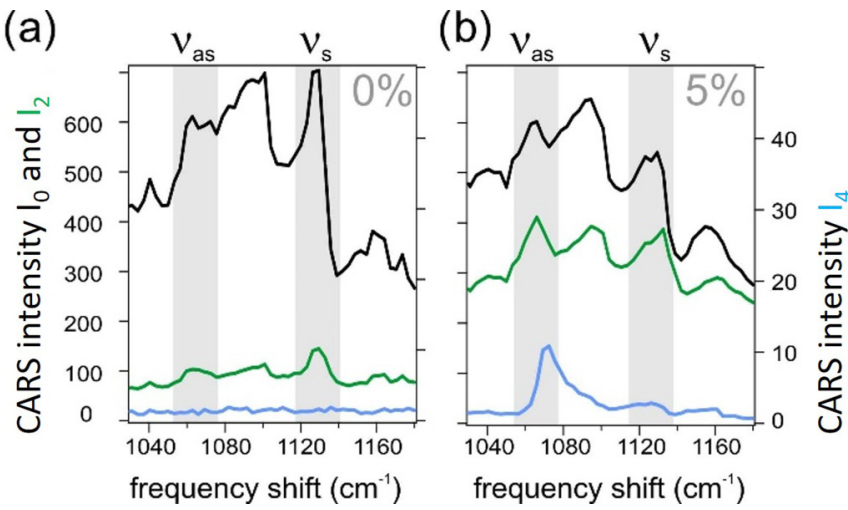

FIG. 4. Symmetry-resolved CARS of multilamellar vesicles in the carbon-carbon bending region. (a) Order 0 (black upper line), 2 (green middle line), and 4 (blue lower line) of MLVs with $0 \%$ of cholesterol. (b) Order 0 (black upper line), 2 (green middle line), and 4 (blue lower line) of MLVs with 5\% of cholesterol.

distribution function [28]. The consequence is an increase of the order 2, even for the nonresonant part, and the presence of order 4 in the distribution function [see Fig. 4(b)]. The order 4 of the symmetric bending is probably weaker which is why we did not see it in our measurement. A higher concentration of cholesterol should increase the order- 4 contributions further, however, higher concentration of cholesterol also results in a stronger birefringence of the MLV and makes the observation impossible [28].

\section{SUMMARY AND DISCUSSION}

The spherical formalism described here allows to reduce the tedious calculation associated with the Cartesian formalism. With the spherical formalism, one can express the different spherical components of a specific vibration symmetry from the Raman susceptibility tensor expressed into the spherical basis. Then, the spherical components of the CARS tensor are directly calculated. Using a spherical approach for the measurement allows to read out directly the symmetry of the macroscopic susceptibility with a single polarization set. In crystalline sample, the spherical components of the CARS tensor, built from the Raman spherical components, allow to predict and measure the symmetry of vibrations. However, the CARS tensor, due to its construction, does not contain more information on the symmetry vibration than the Raman tensor, even if the spherical components available have increased to $m=4$.

In soft matter, the spherical components of the macroscopic susceptibility allows to measure the distribution function coefficients and the spherical coefficients of the microscopic susceptibility. In this case, the symmetry of the macroscopic susceptibility comes from either the microscopic susceptibility symmetry or the distribution molecular function symmetry. The assignment of each contribution is difficult to perform without any assumption on the sample, either the molecular symmetry or the distribution of molecules. However, a strong advantage occurs with CARS with respect to Raman: the high-rank CARS tensor allows to reveal the fourth order of the distribution function [28]. Providing that the molecular susceptibility contains a nonvanishing order 4 , the fourth order of the molecular distribution function is available and enriches the information about the molecular distribution.

The building process of the macroscopic susceptibility, from the matching symmetry between the microscopic susceptibility and the distribution function, has a strong and unknown consequence on the polarization measurements and their interpretation. Usually, the assumption to retrieve the molecule orientation with CARS $[6,28]$, third harmonic generation [30] or second harmonic generation [5], is based on bond additive model. The bond additive model consists on making the assumption that only one Cartesian component is nonvanishing. Then, the elementary tensor is distributed along the molecular distribution in order to generate a macroscopic susceptibility tensor. With the spherical formalism, a molecular tensor with only one nonvanishing Cartesian component leads to a molecular tensor with several spherical components, according to Table I. In this case, the macroscopic tensor is not filtered out by the molecular tensor. Consequently, the distribution function can be read out efficiently. This assumption can be done for uniaxial bonds of group $C_{n}$, like lipid molecules. However, if the molecular tensor does not possess some orders, these missing orders will not appear into the macroscopic susceptibility and the distribution function will be misinterpreted. In this case, the symmetry of the vibration must be taken into account.

Finally, the spherical formalism in polarized nonlinear optics provides quantitative molecular bond symmetry imaging, without the need for any polarizer rotation nor signal processing. This approach, operated in microscopy, should open new possibilities for chemical and biochemical applications where the molecular organization is involved.

\section{ACKNOWLEDGMENTS}

The authors acknowledge financial support from the Centre National de la Recherche Scientifique (CNRS), Aix Marseille University A*Midex (Grant No. ANR-11-IDEX-000102). This work has been supported by Contracts FranceBioImaging (Grant No. ANR-10-INBS-04-01), France Life Imaging (Grant No. ANR-11-INSB-0006), and Tremplin-ERC (Grant No. ANR-16-TERC-0008-01).

\section{APPENDIX}

The irreducible components $\gamma_{m}^{l}$ of order $l$ and $m$ are connected to the $\gamma_{i j k l}$ components according to the following relation:

$$
\begin{aligned}
\gamma_{m}^{l}= & \sum_{h i j k} \sum_{\substack{m_{0}, m_{1} \\
m_{2}, m_{3}}} \sum_{l_{i}^{\prime}, l_{i}}\left(l_{3} m_{3} l_{2} m_{2} \mid l_{i}^{\prime} m_{i}^{\prime}\right) \\
& \times\left(l_{i}^{\prime} m_{i}^{\prime} l_{1} m_{1} \mid l_{i} m_{i}\right)\left(l_{i} m_{i} l_{0} m_{0} \mid l m\right) V_{l_{i}^{\prime}}^{l} U_{l_{i}^{\prime}}^{l_{i}^{\prime}} \\
& \times\left(l_{1} m_{1} \mid h\right)\left(l_{2} m_{2} \mid i\right)\left(l_{3} m_{3} \mid j\right)\left(l_{4} m_{4} \mid k\right) \gamma_{h i j k},
\end{aligned}
$$

where $V_{l_{i}^{\prime}}^{l}$ and $U_{l_{i}}^{l_{i}^{\prime}}$ are the symmetric recoupling matrix [23,31], $\left(l_{2} m_{2} l_{1} m_{1} \mid l_{i} m_{i}\right)$ are the Clebsch-Gordan coefficients, and $(\mathrm{lm} \mid \mathrm{i})$ are the unitary transformation matrix between Cartesian and spherical basis

$$
(\operatorname{lm} \mid i)=\left(\begin{array}{ccc}
i / \sqrt{2} & 1 / \sqrt{2} & 0 \\
0 & 0 & -i \\
-i / \sqrt{2} & 1 / \sqrt{2} & 0
\end{array}\right)
$$


[1] A. Zumbusch, G. R. Holtom, and X. S. Xie, Three-Dimensional Vibrational Imaging by Coherent Anti-Stokes Raman Scattering, Phys. Rev. Lett. 82, 4142 (1999).

[2] W. Min, C. W. Freudiger, S. Lu, and X. S. Xie, Coherent nonlinear optical imaging: Beyond fluorescence microscopy, Annu. Rev. Phys. Chem. 62, 507 (2011).

[3] C. W. Freudiger, W. Min, B. G. Saar, S. Lu, G. R. Holtom, C. He et al., Label-free biomedical imaging with high sensitivity by stimulated Raman scattering microscopy, Science 322, 1857 (2008); P. Berto, E. R. Andresen, and H. Rigneault, BackgroundFree Stimulated Raman Spectroscopy and Microscopy, Phys. Rev. Lett. 112, 053905 (2014); Y. Ozeki, W. Umemura et al., High-speed molecular spectral imaging of tissue with stimulated Raman scattering, Nat. Photonics 6, 845 (2012).

[4] J. L. Oudar, R. W. Smith, and Y. R. Shen, Polarization-sensitive coherent anti-Stokes Raman spectroscopy, Appl. Phys. Lett. 34, 758 (1979).

[5] J. Duboisset, D. Ait-Belkacem, M. Roche, H. Rigneault, and S. Brasselet, Generic model of the molecular orientational distribution probed by polarization-resolved second-harmonic generation, Phys. Rev. A. 85, 043829 (2012).

[6] J. Duboisset, P. Berto, P. Gasecka, F. Z. Bioud, P. Ferrand, H. Rigneault, and S. Brasselet, Molecular orientational order probed by coherent anti-Stokes Raman scattering (CARS) and stimulated Raman scattering (SRS) microscopy: A spectral comparative study, J. Phys. Chem. B 119, 3242 (2015).

[7] G. De Vito, A. Canta, P. Marmiroli, and V. Piazza, A large-field polarization-resolved laser scanning microscope: applications to CARS imaging, J. Microscopy 260, 194 (2015)

[8] X. Y. Dow, E. L. DeWalt, J. A. Newman, C. M. Dettmar, and G. J. Simpson, Unified theory for polarization analysis in second harmonic and sum frequency microscopy, Biophys. J. 111, 1553 (2016).

[9] J. H. Christie and D. J. Lockwood, Selection rules for threeand four-photon Raman interactions, J. Chem. Phys. 54, 1141 (1971); Z. Ożgo and S. Kielich, Coordinates for the description of harmonic generation of circularly polarized laser light, Phys. C (Amsterdam) 81, 151 (1976).

[10] R. P. Davies, A. J. Moad, G. Scott Goeken, R. D. Wampler, and G. J. Simpson, Selection rules and symmetry relations for four-wave mixing measurments of uniaxial assemblies, J. Phys. Chem. B 112, 5834 (2008).

[11] F. Munhoz, H. Rigneault, and S. Brasselet, High Order Symmetry Structural Properties of Vibrational Resonances Using Multiple-Field Polarization Coherent Anti-Stokes Raman Spectroscopy Microscopy, Phys. Rev. Lett. 105, 123903 (2010).

[12] J. Jerphagnon, D. Chemla, and R. Bonneville, The description of the physical properties of condensed matter using irreducible tensors, Adv. Phys. 27, 609 (1978).

[13] J. Zyss, Molecular engineering implications of rotational invariance in quadratic nonlinear optics: From dipolar to octupolar molecules and materials, J. Chem. Phys. 98, 6583 (1993).

[14] S. Brasselet and J. Zyss, Multipolar molecules and multipolar fields: probing and controlling the tensorial nature of nonlinear molecular media, J. Opt. Soc. Am. B 15, 257 (1998).

[15] S.-H. Lee, J. Wang, S. Krimm, and Z. Chen, Irreducible representation and projection operator application to understanding nonlinear optical phenomena: Hyper-Raman, sum frequancy generation, and four-wave mixing spectroscopy, J. Phys. Chem. A 110, 7035 (2006).

[16] B. F. Gachter, The irreducible Raman scattering tensor operator for the 32 crystallographic point groups and its application to the resonance and electronic Raman effect, J. Mol. Spec. 63, 1 (1976).

[17] C. Cleff, A. Gasecka, P. Ferrand, H. Rigneault, S. Brasselet, and J. Duboisset, Direct imaging of molecular symmetry by coherent anti-Stokes Raman scattering, Nat. Commun. 7, 11562 (2016).

[18] P. D. Maker, Spectral broadening of elastic second-harmonic light scattering in liquids, Phys. Rev. A 1, 923 (1970).

[19] M. A. Yuratich and D. C. Hanna, Coherent anti-Stokes Raman spectroscopy (CARS), Mol. Phys. 33, 671 (1977).

[20] S. Rahav and S. Mukamel, Stimulated coherent anti-Stokes Raman spectroscopy (CARS) resonances originate from doubleslit interference of two-photon Stokes pathways, Proc. Natl. Acad. Sci. USA 107, 4825 (2010).

[21] S. L. Altmann and A. P. Cracknell, Lattice harmonics I cubic group, Rev. Mod. Phys. 37, 19 (1965).

[22] N. Baddour, Operational and convolution properties of threedimensional Fourier transforms in spherical coordinates, J. Opt. Soc. Am. A 27, 2144 (2010).

[23] J. Duboisset, H. Rigneault, and S. Brasselet, Filtering of matter symmetry properties by circularly polarized nonlinear optics, Phys. Rev. A 90, 063827 (2014).

[24] R. Bonneville and D. S. Chemla, Symmetries of the light scattering processes in low-density fluids, Phys. Rev. A 17, 2046 (1978).

[25] S. Brustlein, P. Ferrand, N. Walther, S. Brasselet, C. Billaudeau, D. Marguet et al., Optical parametric oscillator-based light source for coherent Raman scattering microscopy: practical overview, J. Biomed. Opt. 16, 021106 (2011).

[26] P. Ferrand, GPScan.VI: A general-purpose LabVIEW program for scanning imaging or any application requiring synchronous analog voltage generation and data acquisition, Comput. Phys. Commun. 192, 342 (2015).

[27] C. Marcolli, P. Laine, R. Bühler, G. Calzaferri, and J. Tomkinson, Vibrations of H8Si8O12, D8Si8O12, and H10Si10O15 as determined by INS, IR, and Raman experiments, J. Phys. Chem. B 101, 1171 (1997).

[28] F. Z. Bioud, P. Gasecka, P. Ferrand, R. Rigneault, J. Duboisset, and S. Brasselet, Structure of molecular packing probed by polarization-resolved nonlinear four-wave mixing and coherent anti-Stokes Raman-scattering microscopy, Phys. Rev. A 89, 013836 (2014).

[29] M. Harrand, Polarized Raman spectra of oriented dipalmitoylphosphatidylcholine (DPPC). I. Scattering activities of skeletal stretching and methylene vibrations of hydrocarbon chains, J. Chem. Phys. 79, 5639 (1983).

[30] M. Zimmerley, P. Mahou, D. Débarre, M.-C. Schanne-Klein, and E. Beaurepaire, Probing Ordered Lipid Assemblies with Polarized Third-Harmonic-Generation Microscopy, Phys. Rev. X 3, 011002 (2013).

[31] H. A. Jahn, and H. Van Wieringen, Theoretical studies in nuclear structure. IV. wave functions for the nuclear $\mathrm{p}$-shell Part A. $\left\langle p^{n} \mid p^{n-1} p\right\rangle$ fractional parentage coefficients, Proc. R. Soc. London A 209, 502 (1951). 